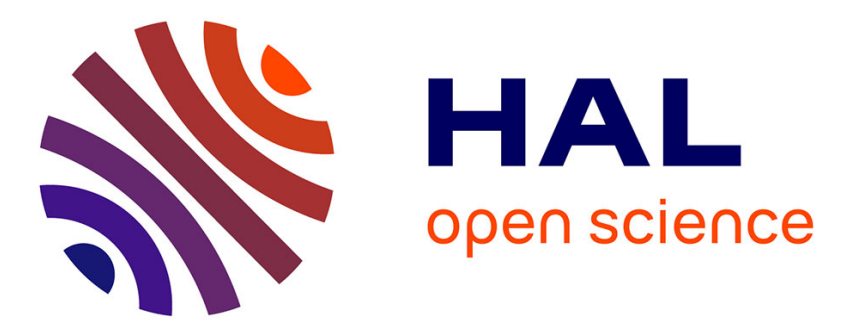

\title{
Small angle neutron scattering study on a phase separation in a 3-component microemulsion system
}

H. Seto, E. Yokoi, S. Komura, D. Schwahn, K. Mortensen, J. Suzuki, M. Ohnuma, Y. Ito

\section{- To cite this version:}

H. Seto, E. Yokoi, S. Komura, D. Schwahn, K. Mortensen, et al.. Small angle neutron scattering study on a phase separation in a 3-component microemulsion system. Journal de Physique IV Proceedings, 1993, 03 (C8), pp.C8-161-C8-164. 10.1051/jp4:1993831 • jpa-00252262

\section{HAL Id: jpa-00252262 https://hal.science/jpa-00252262}

Submitted on 1 Jan 1993

HAL is a multi-disciplinary open access archive for the deposit and dissemination of scientific research documents, whether they are published or not. The documents may come from teaching and research institutions in France or abroad, or from public or private research centers.
L'archive ouverte pluridisciplinaire HAL, est destinée au dépôt et à la diffusion de documents scientifiques de niveau recherche, publiés ou non, émanant des établissements d'enseignement et de recherche français ou étrangers, des laboratoires publics ou privés. 


\title{
Small angle neutron scattering study on a phase separation in a 3-component microemulsion system
}

\author{
H. SETO, E. YOKOI, S. KOMURA, D. SCHWAHN*, K. MORTENSEN**, J. SUZUKI***, \\ M. OHNUMA ${ }^{* * *}$ and Y. ITO ${ }^{* * * *}$
}

FLAS, Hiroshima University, Higashihiroshima 724, Japan

${ }^{*}$ IFF, KFA Jülich, 5170 Jülich, Germany

** Ris $\emptyset$ National Laboratory, 4000 Roskilde, Denmark

*** Department of Neutron Scattering, JAERI, Tokai 319-11, Japan

**** ISSP, University of Tokyo, Roppongi, Tokyo 106, Japan

\begin{abstract}
In literature, the 3-component microemulsion system consisting of AOT, water and $n$ decane is known to belong to 3D-Ising universality class so far. Recently, we have found that the critical exponent of the susceptibility is the meanfield value at nearcritical region, and at the same time we have observed a small change of the water droplet size approaching a phase separation point. In this work, further small angle neutron scattering results are presented obtained at the new SANS equipment in JAERI. The evidences described above were confirmed and a detailed phase diagram has been obtained, but the critical point was not found. These results seem to suggest that the change of the droplet structure on approaching the critical point affects the critical point itself.
\end{abstract}

\section{INTRODUCTION}

At room temperature, the oil rich mixture of AOT (2-ethylhexylsulfosuccinate), water and n-decane forms "water-in-oil" microemulsion, that is, water droplets coated with AOT film are dispersed in n-decane. The droplet size was supposed to be constant and monodisperse when the temperature or concentration is varied, provided that the water to AOT ratio is kept constant. With increasing temperature, the system decomposes into two phases, namely droplet rich and poor domains. Below the decomposition temperature, a density fluctuation of the droplets occurs and becomes stronger with increasing temperature. It determines the critical phenomena near the critical temperature $T_{\mathrm{c}}{ }^{1,2)}$ Several authors ${ }^{2-6)}$ have investigated this phenomenon by means of small angle neutron scattering (SANS) or light scattering (LS). As summarized by Rouch et al., ${ }^{6}$ ) the trend of interpretation of the observed critical exponents has been that of 3D-Ising.

Recently, we have examined the critical behavior by means of small angle neutron scattering at RIS $\emptyset$ National Laboratory, and observed the meanfield value for the critical exponent of the susceptibility at "near-critical region". At the same time we found that the droplets are rather mono-disperse and that their size decreases approaching the phase separation point. From the temperature dependence of the susceptibility, we have determined the spinodal points and the binodal points for 4 compositions.

In order to explain the obtained phase diagram, the Gibbs' free energy of the van der Waals model was introduced because this model is equivalent to a meanfield approximation of interacting particles. In dimensionless form, the free energy reads,

$$
f \equiv \frac{F}{(V / \Omega) k_{B} T}=-\phi\left\{\ln \Omega\left(\frac{1}{\dot{\phi}}-b^{\prime}\right)+c(T)\right\}-\phi^{2} \chi(\phi, T)
$$

with the order parameter $\phi$, which is the volume fraction of droplets, $\Omega \equiv \phi V / N, i$. e., the volume assigned to each particle, and $b^{\prime} \equiv b / \Omega$, an effective volume factor of each particle. 
$c(T)$ includes all $\phi$-independent terms and $\chi(\phi, T)=a / \Omega k_{\mathrm{B}} T$ is the Flory-Huggins parameter. The spinodal points were fitted by the formula

$$
T_{\mathrm{s}}=\frac{-1322-72920 \phi+349900 \phi^{2}}{1 /\left(2 \phi\left(1-\phi b^{\prime}\right)^{2}\right)-18.63-7.840 \phi-242.4 \phi^{2}},
$$

assuming the 2 nd polynomial dependence on $\phi$ for the Flory-Huggins parameter. The binodal points could also be explained by this model rather well.7)

In order to confirm and to develop the results observed before at RIS $\emptyset$, further experiments with other concentrations were performed at the new SANS spectrometers, SANS-U and SANS-J at JRR-3M in JAERI. As a result, the meanfield behavior for the susceptibility and the temperature dependence of the Guinier radius could be reproduced, but no critical concentration could be determined. ${ }^{8)}$

\section{EXPERIMENTAL}

All the samples were prepared at the same water-to-AOT ratio as the $3 / 5 / 95$ composition microemulsion, which is the mixture of $3 \mathrm{~g}$ of AOT, $5 \mathrm{ml}$ of water and $95 \mathrm{ml}$ of $\mathrm{n}$ decane. ${ }^{2)}$ The volume fraction $\phi$ of both the surfactant and water mixture against the $n$-decane is the order parameter, because it is proportional to the droplet density in the whole system. The $98 \%$ AOT purchased from the Aldrich Chemical Company, $99.9 \%$ heavy water supplied by Isotec Inc. and the $99 \%$ n-decane by Katayama Chemical were used without any further treatments. The mixtures were in a niobium cell with $1 \mathrm{~mm}$ thick quartz-window during the SANS experiments. All the samples were mixed by the dilution method from a batch of $\phi=$ 0.2 .

The SANS measurements were carried out with $6.4 \AA$ and $7 \AA$ incident neutron beams, taken from the cold neutron source and monochromatized a by mechanical velocity selector. Observed momentum transfer ranges over $3.0 \times 10^{-3} \leq Q \leq 7.8 \times 10^{-2}\left[\AA^{-1}\right]$ for SANS-J and $4.2 \times 10^{-3} \leq Q \leq 3.1 \times 10^{-2}\left[\AA^{-1}\right]$ for SANS-U, and the wavelength resolutions were $13 \%$ and $10 \%$, respectively. The multi-wire 2-dimensional position sensitive proportional counters were placed at 3,10 and $12 \mathrm{~m}$ apart from the sample position. The raw data were radially integrated and calibrated into differential cross sections $d \Sigma / d \Omega$ using the incoherent scattering from a cell

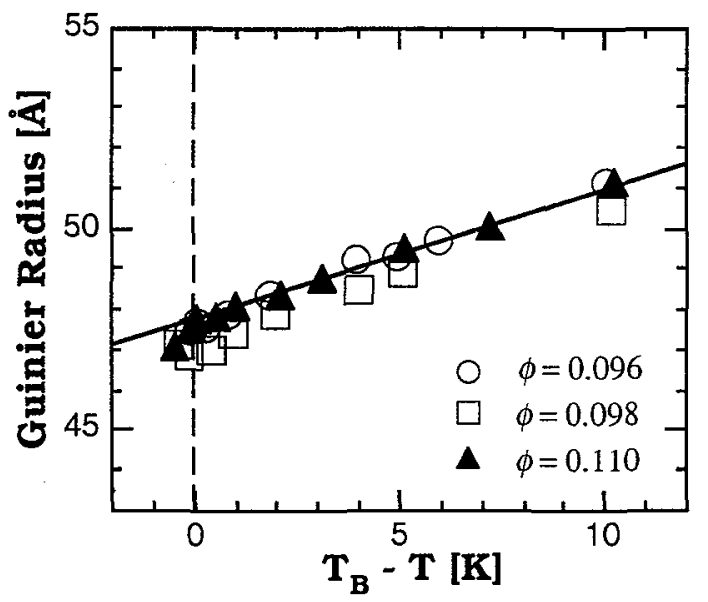

Fig. 1 Temperature dependence of the Guinier radius for $\phi=$ $0.096,0.098$ and 0.110 is shown. The solid straight line is a guide for eyes, and the dashed line is the observed binodal point. The horizontal axis indicates the temperature deference from the binodal points. filled with light water. The sample cell was placed in an electric furnace controlled within $0.003 \mathrm{~K}$ by two platinum resister temperature sensors and a PID-type control program running in a personal computer.9)

\section{EXPERIMENTAL RESULTS}

In Fig. 1, the temperature dependence of the observed Guinier radius $R_{\mathrm{g}}$ for 3 compositions are shown. It linearly depends upon the temperature for almost all the temperature region. However, a slight deviation from the straight line is found near the binodal point. The temperature variations of the scattering cross sections at relatively high- $Q$ region are shown in Fig. 2. Below the phase separation temperature, the profiles at high- $Q$ region are about the same and follow the Porod's law, that is, proportional to $Q^{-4}$, but above the binodal point, an appreciable change of the cross section is found. This 


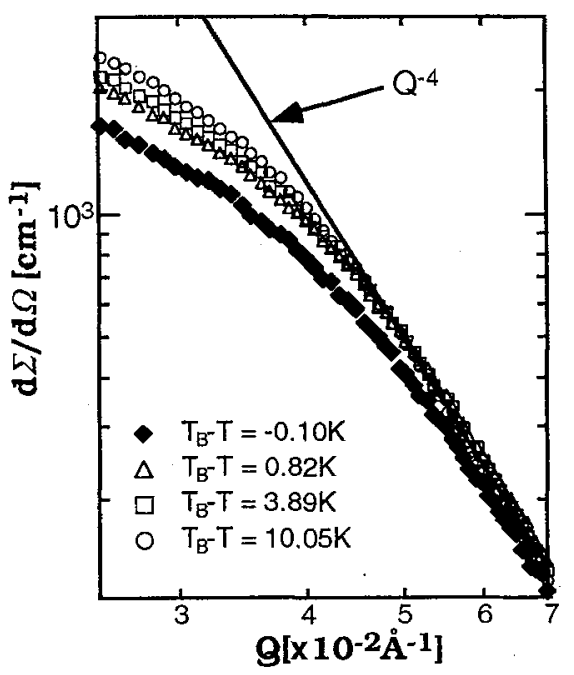

Fig.2 Temperature variation of the scattering profiles for $\phi=0.096$ in high $Q Q$ region in double logarithmic plot is displayed. The solid line is proportional to the $Q^{-4}$ dependence.

experimental evidence suggests that the size and/or shape of droplets changes on approaching and crossing the phase separation point, and the structure of droplets may be influenced by the strong fluctuations near phase separation.

The structure factor $S(Q)$ was obtained using the temperature dependent form factor of the droplet. The structure factor of the system follows the Ornstein-Zernike formula in low- $Q$ region as observed from the Zimm plot, from which the forward scattering $S_{0}$ and the correlation length $\xi$ were obtained. In Fig. 3, the observed temperature dependence of the inverse forward scattering $S_{0}{ }^{-1}$ for $\phi=0.096,0.098$ and 0.110 are shown. The inverse forward scattering is found to be proportional to $1 / T$. This means that the critical exponent $\gamma$ for the susceptibility $\chi$ is about 1.0 , which is the meanfield value. These results confirm the evidences obtained at RIS $\emptyset$. The spinodal point could be obtained from the intercept of these fitted straight lines on the abscissa, and the binodal point is also obtained from the 'kink', where $S_{0}$ stops to diverge. The observed spinodal points and the binodal points for the range $\phi=0.092 \sim 0.099$ and the fitted binodal and spinodal lines are given in Fig. 4. Although we measured many concentrations around the theoretically expected critical point, we could not find a point where the spinodal and binodal coincide with each other.
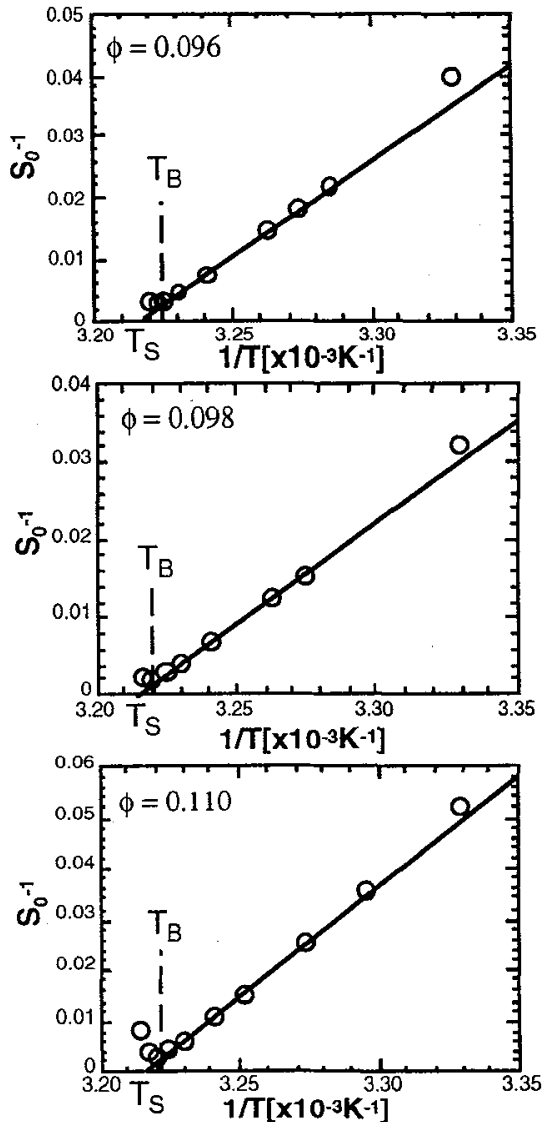

Fig. 3 Temperature dependence of the susceptibility for $\phi=0.096,0.098$ and 0.110 are given. The meanfield behavior was confirmed and both spinodal and binodal points were obtained.

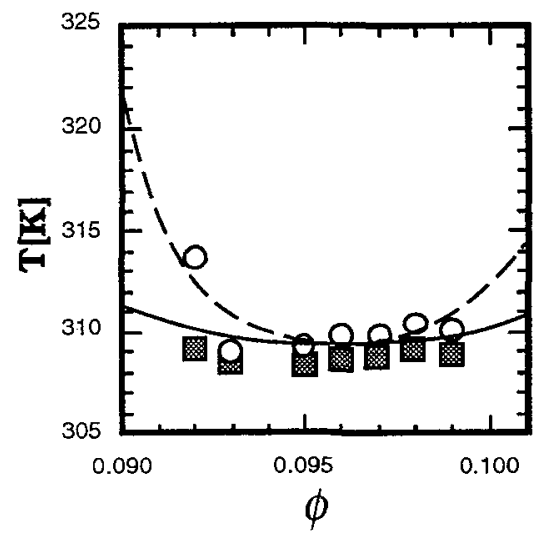

Fig. 4 The phase diagram obtained at JAERI. The open circles and the hatched squares indicate the observed spinodal points and binodal points, respectively, and the dashed and the solid lines are the calculated spinodal and binodal lines. 


\section{DISCUSSION AND CONCLUSION}

In this work, the meanfield behavior of the susceptibility of density fluctuation of water droplets in near-critical region is confirmed again for various concentrations. Within our results, the critical point was not observed. We believe that this evidence is not because of an inadequacy of our experimental results but is inherent of the system. From the Guinier radius, the droplet size decreasing with increasing temperature below the decomposition point, and seems to be affected very much by the critical fluctuations near the phase boundary where a stronger decrease of $R_{\mathrm{g}}$ is observed (see Fig. 1). Thus there is a possibility that the rearrangement of droplets occurs before reaching the theoretically predicted binodal points since the fluctuation affects the form of the droplets.

\section{ACKNOWLEDGMENTS}

This work is supported by Yamada Science Foundation, Nissan Science Foundation and a Grant-in-Aid for Scientific Research (No. 04740200) from the Japanese Ministry of Education.

\section{REFERENCES}

[1] For a general survey, see "Micellar Solutions and Microemulsions", CHEN, S. -H. and RAJAGOPALAN, eds., Springer-Verlag, New York, 1990.

[2] HUANG, J. S. and KIM, M. W., Phys. Rev. Lett. 47 (1981) 1462.

[3] KOTLARCHYK, M., CHEN, S. -H. and HUANG J. S., Phys. Rev. A28 (1983) 508.

[4] KOTLARCHYK, M., CHEN, S. -H., HUANG J. S. and KIM, M. W., Phys. Rev. A29 (1984) 2054.

[5] HONORAT, P., ROUX, D. and BELLOCQ, A. M., J. Phys. Lett. 45 (1984) L-961.

[6] ROUCH J., SAFOUANE, A., TARTAGlia, P. and CHEN, S. -H., J. Chem. Phys. 90 (1989) 3756.

[7] The details will be published in J. Chem. Phys. 99 (1993).

[8] A part of the results will be published in the proceedings of "5th International Symposium on Advanced Nuclear Energy Research" (1993).

[9] The temperature control program was developed by Y. Noda at Chiba University. 